\title{
The morpho/functional discrepancy in the cerebellar cortex: looks alone are deceptive
}

\author{
Dan Rokni ${ }^{*}$, Rodolfo Llinas ${ }^{2}$ and Yosef Yarom ${ }^{1}$ \\ 1 Institute of Life Sciences, and Interdisciplinary Center for Neural Computation, Hebrew University, Jerusalem, Israel \\ 2 Department of Physiology and Neuroscience, New York University School of Medicine, New York, NY, USA
}

Edited by:

Ranulfo Romo, Universidad Nacional Autónoma de México, Mexico

Reviewed by:

Chris de Zeeuw, Department of

Neuroscience, Erasmus Medical Center, Rotterdam, The Netherlands

Henry Markram, Brain Mind Institute, Ecole Polytechnique Fédérale

de Lausanne, Switzerland

* Correspondence:

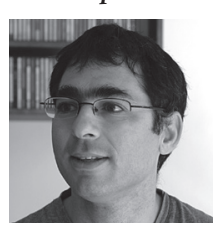

Dan Rokni is completing his $P h D$ dissertation to be submitted to the Hebrew University of Jerusalem. In his research, which focuses on the cerebellar cortex, he combines electrophysiological and imaging techniques in a goal directed effort to unravel the functional local circuitry. dan.rokni@mail.huji.ac.il

In a recent report we demonstrated that stimulation of cerebellar mossy fibers synchronously activates Purkinje cells that are located directly above the site of stimulation. We found that the activated Purkinje cells are arranged in a radial patch on the cerebellar surface and that this organization is independent of the integrity of the inhibitory system. This arrangement of activity is counterintuitive. The anatomical structure with the extensive parallel fiber system implies that mossy fiber stimulation will activate Purkinje cells along a beam of parallel fibers. In this short review we highlight this discrepancy between anatomical structure and functional dynamics and suggest a plausible underlying mechanism.

Keywords: climbing fiber, mossy fiber, Purkinje cell, parallel fibers

\section{INTRODUCTION}

The cerebellum, with its extraordinarily stereotyped morphology, has been hailed as the epitome for morphology based neuronal circuit analysis. Indeed, the striking geometrical collimation between the isoplanar Purkinje cell dendrites and the beams of parallel fibers is nothing short of astonishing. The intuitive view emerging from this unusual geometry is that mossy fibers that activate the parallel fiber system, will activate Purkinje cells sequentially, enabling the generation of accurate time intervals. This intuitive view has been the basis for theories of cerebellar function that will be reviewed below. In contrast, physiological evidence point to a radial organization of the mossy fiber input, i.e. patches of Purkinje cells are synchronously activated. This apparent discrepancy between anatomical structure and functional dynamics is the focus of this short review. We will briefly describe the cytoarchitecture of the cerebellar cortex and summarize morphologically inspired theories of cerebellar function. We will then focus on physiological evidence for the radial organization of the mossy fiber input and its functional implications.

\section{THE CYTOARCHITECTURE OF THE CEREBELLAR CORTEX}

The cerebellar cortex is a continuous sheet of repeating neuronal networks elongated in the rostro-caudal direction and folded in an accordion like fashion into folia for space conservation reasons. Its cytological architecture has been extensively studied and is summarized in the seminal work of Palay and Chan-Palay (1974). The most striking architectural feature of the cerebellar cortex is the orthogonal organization of almost all its neuronal elements. Purkinje cells, molecular layer interneurons, and climbing fibers are all oriented in parasagittal planes, while the parallel fibers are oriented in the medio-lateral axis. The preservation of this architecture through vertebrate evolution suggests that it is of outmost importance, either functionally, or for packing efficiency. Classically, the cerebellar cortex is divided into three layers: the superficial molecular layer, the deep granule cell layer and between them a single cell deep Purkinje cell layer (Figure 1). The latter is composed of the somata of the principal elements of the cerebellar cortex: 


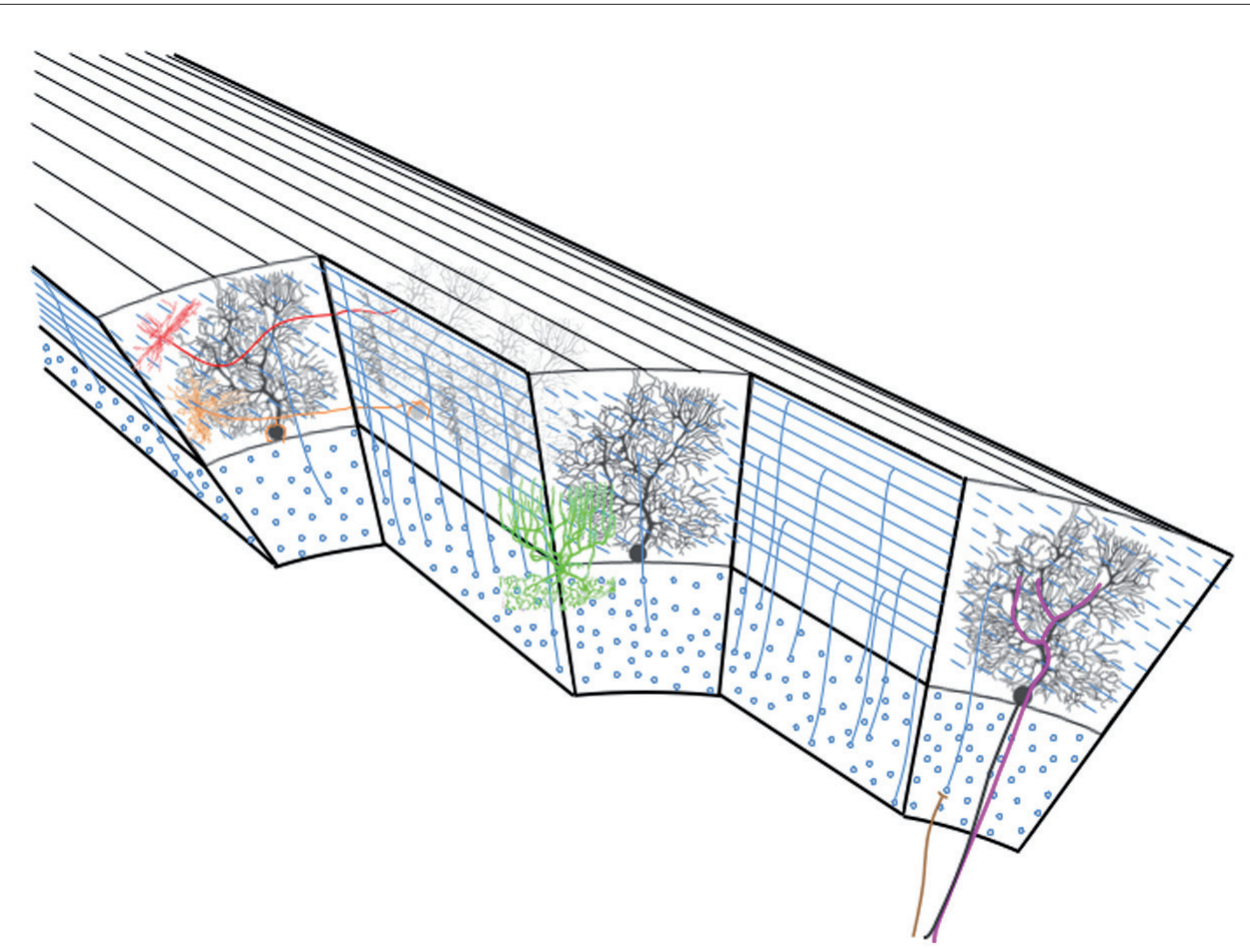

Figure 1 | The cytoarchitecture of the cerebellar cortex. A parasagittal cut through the cerebellar cortex shows the arrangement of the cell types. Black - Purkinje cells. Blue - granule cells. Green - golgi cell. Red - stellate cell. Orange - basket cell. Mossy and climbing fibers are shown in brown and magenta, respectively.

the Purkinje cells (Figure 1, black). The axons of these strangely flat neurons, whose dendrites are organized in parasagittal planes next to each other as pages in a book, are the sole output of the cerebellar cortex.

Two main types of input reach the cerebellar cortex: The mossy fibers and the climbing fibers. The numerous mossy fibers (Figure 1, brown) that originate in both lower and higher levels of the neuraxis innervate the granule cells (Figure 1, red). Granule cell axons ascend through the cerebellar cortex and then bifurcate to form the parallel fiber system. These fibers run about $5 \mathrm{~mm}$ (Brand et al., 1976; Harvey and Napper, 1988; Mugnaini, 1983) along the medio-lateral axis of the cerebellar cortex forming en-passant excitatory connections with about 600 Purkinje cell dendrites (Harvey and Napper, 1988). About 200,000 parallel fibers cross the dendritic field of every Purkinje cell, each establishing a single synaptic connection (Napper and Harvey, 1988). Climbing fibers (Figure 1, magenta) originate exclusively in the inferior olivary nucleus and terminate directly on Purkinje cells and also on Golgi cells (Shinoda et al., 2000). Each Purkinje cell is innervated by a single climbing fiber that establishes multiple synaptic contacts with the lower two-thirds of its dendrite. The climbing fiber system is organized parasagittally both in the macro and in the micro levels. At the micro level it is structured to fit the Purkinje cell dendrites, while at the macro level all collaterals of a single olivary neuron are restricted to a narrow rostrocaudal plane covering many folia.

Two types of inhibitory interneurons are located within the cerebellar molecular layer: the stellate (Figure 1, red) and basket cells (Figure 1, orange). They form a chemically and electrically interconnected network that receives excitatory input from granule cell axons (Mann-Metzer and Yarom, 1999; Sotelo and Llinas, 1972). Their axons run along the rostro-caudal axis and inhibit Purkinje cell dendrites (stellate) and somata (basket).

The third type of cerebellar inhibitory interneuron is the Golgi cells (Figure 1, green) whose somata lie in the upper granule cell layer. They receive input from mossy fibers, both directly, and through granule cell axons, and inhibit mossy fiber-granule cell inputs at their junction, forming a complex glomerulus (knot). These are the only three dimensional neurons in the cerebellar cortex, i.e. their dendrites and axons extend both rostro-caudally, and medio-laterally forming 
radial cylindrical dendritic and axonic trees, in the molecular and granule cell layers respectively.

Two other types of cerebellar cortical neurons that have been more recently incorporated into the classical cerebellar cortex circuit are the excitatory unipolar brush cells and the inhibitory Lugaro cells, both of which are located within the granular layer. Unipolar brush cells are mostly found in the vestibulocerebellum and mediate interactions between mossy fibers and granule cells. Lugaro cells receive mossy fiber input and inhibit Golgi cells.

It is the remarkable conserved orthogonal arrangement of the parasagittal Purkinje cell dendrites and the coronal parallel fibers that prompted cytoarchitecture-based theories on the function of the cerebellar cortex.

\section{ANATOMY-INSPIRED THEORIES OF CEREBELLAR FUNCTION}

Various theories of cerebellar function and its mode of operation have been proposed in past and present decades (Albus, 1971; Braitenberg and Atwood, 1958; Marr, 1969; Ohyama et al., 2003; Thach et al., 1992; Wolpert et al., 1998). Although the anatomy of the cerebellar cortex subserved as a building block in all of these theories, only a subset of them can be regarded as anatomically inspired theories, where a major role was attributed to the unique relationships between the parallel fibers and Purkinje cells. Some accredited parallel fibers the role of maximizing the number of possible connections between granule cells and Purkinje cells (Albus, 1971; Marr, 1969; Thach et al., 1992), while others emphasized the sequential activation of Purkinje cells, as a possible mechanism to generate accurate time intervals (Braitenberg and Atwood, 1958). Three examples of such theories are shortly reviewed below.

Marr (1969), in his seminal work, suggested that the cerebellar cortex anatomy enables it to discriminate between similar input patterns. In his view the divergence of the mossy fiber input in the granule cell layer and the convergence of granule cell axons on single Purkinje cells increase the dimensionality of the mossy fiber input onto Purkinje cells and thus can increase the differences between similar mossy fiber inputs and facilitate differential responses. Here the emphasis is on maximizing the convergence of granule cells onto Purkinje cells that is brought about by the parallel fibers.

Thach et al. (1992) assumed that Purkinje cells that are organized along the medio-lateral axis control motor behavior of different body parts. In order to perform a coordinated movement a common input to these cells is needed. It was sug- gested that this common input is provided by the parallel fibers. Complementary to Marr's theory, here the authors emphasized the divergence of single granule cell axons onto many Purkinje cells.

Braitenberg and Atwood (1958) stated that the unique anatomy of the cerebellar cortex suggests that it serves as a transformer of spatial into temporal patterns. Action potentials that propagate along the parallel fibers will, according to this view, sequentially activate Purkinje cells with accurate time intervals and thus the distance travelled by action potentials will be coded by the time of the Purkinje cell response. After acknowledging the difficulties in finding sequential activation of Purkinje cells (as will be reviewed below), Braitenberg formulated the "tidal wave" theory. The cerebellar cortex was now attributed the role of transforming spatio-temporal patterns of mossy fiber input into spatio-temporal patterns of Purkinje cell output (Braitenberg et al., 1997). The main idea of the modified hypothesis was that in order to activate a significant number of the parallel fibers that impinge on a specific Purkinje cell, it is necessary to activate many groups of granule cells with time intervals that fit parallel fiber conduction velocity. In its most simplistic form this type of input could code for a movement of a stimulus along a body part.

As stated, the above theories all derive from the geometrical arrangements of the neuronal elements in the cerebellar cortex. Indeed Marr's theory, as well as the early form of Braitenberg's theory was formulated at a time when knowledge about the physiological properties of cerebellar neurons were rather limited. Most importantly, the properties of parallel fiber - Purkinje cell synapses and their spatial organization were scarcely described.

\section{FUNCTIONAL ORGANIZATION OF THE MOSSY FIBER INPUT TO THE CEREBELLAR CORTEX}

The beam like activity of Purkinje cells evoked by parallel fibers was initially supported by numerous studies where surface stimulation directly activated the parallel fibers (Chen et al., 1998; Cohen and Yarom, 1999; Eccles et al., 1966). However, activating the mossy fiber system, either directly or by peripheral stimulus, failed to invoke beams of Purkinje cell activity (Bower and Woolston, 1983; Shambes et al., 1978) but see Garwicz and Andersson (1992). Furthermore, even the expected correlations between Purkinje cells located along the parallel fibers could not be detected (Ebner and Bloedel, 1981; Eccles et al., 1972; Heck et al., 2007), but see Bell and Grimm (1969). In all these studies extracellular record- 
ings of Purkinje cell spiking activity were used. This technique severely limits the characterization of the spatial organization of synaptic input. First, spatial resolution is limited by the distance between recording electrodes. Second, the inability to detect subthreshold activity confines the conclusions to synapses that are strong enough to generate suprathreshold activity. More recently, the use of voltage sensitive dye imaging was implemented in an attempt to overcome these limitations (Cohen and Yarom, 1998, 1999, 2000; Rokni et al., 2008). In our previous publications (Cohen and Yarom, 1998; Rokni et al., 2008) we used this technique in combination with the isolated cerebellar preparation (Llinas et al., 1981). This experimental system provides a much finer spatial resolution, it is extremely sensitive to subthreshold postsynaptic activity, and it greatly simplifies experiments, while preserving the three dimensional structure of the cerebellum (Cohen and Yarom, 1999). Using this technique we demonstrated that direct stimulation of mossy fibers at the level of the white matter, activates Purkinje cells that lie in radial patches of cerebellar cortex (Figure 2A). Postsynaptic activity that propagates along the parallel fiber axis could readily be recorded when parallel fibers were directly activated at the surface of the cerebellar cortex (Figure 2B). These results, which provided direct support for the radial organization of cerebellar cortical response to mossy fiber input as proposed by Llinas (1982), are in sharp contrast to what is expected from the anatomy. Hence, although the anatomical structure is most certainly a crucial component in our understanding of any brain region, a lesson from the cerebellum teaches us it is not enough - looks alone can be deceptive.

Two mechanisms were suggested to resolve the mossy fiber morpho/functional discrepancy. Llinas (1982) suggested that the major granular input onto Purkinje cells is provided by the ascending portion of granule cell axons and not by the parallel fibers. According to this view the mossy fiber input into the cerebellar cortex is most effective when it activates the ascending portion
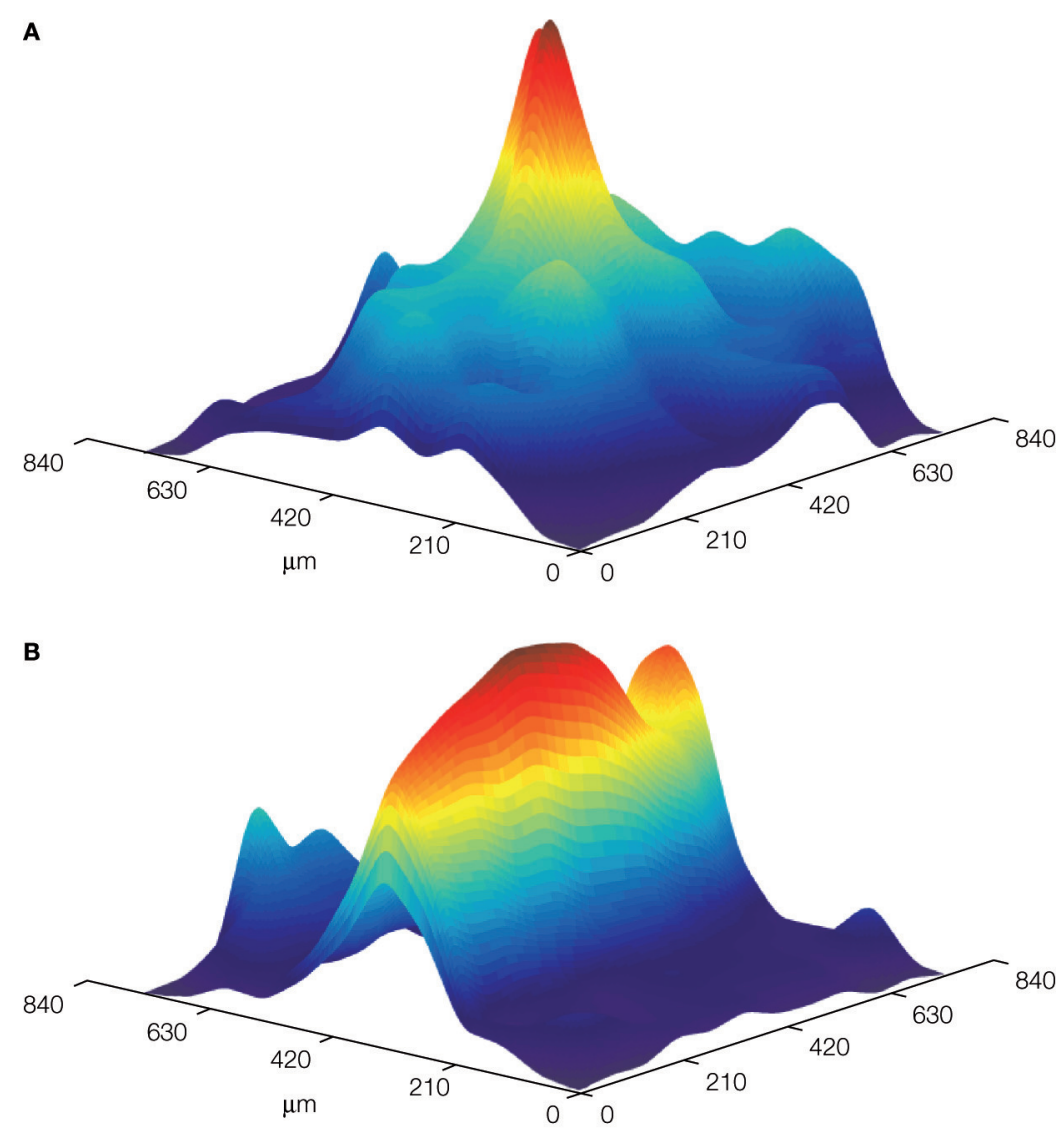

Figure 2 | Color coded surface presentations showing the spatial organization of cerebellar cortical responses to mossy (A) and parallel (B) fiber stimulation. Data obtained from the isolated Guinea pig cerebellum using voltage sensitive dye imaging. Responses were recorded with a 128 photodiode array, and spatially filtered. 


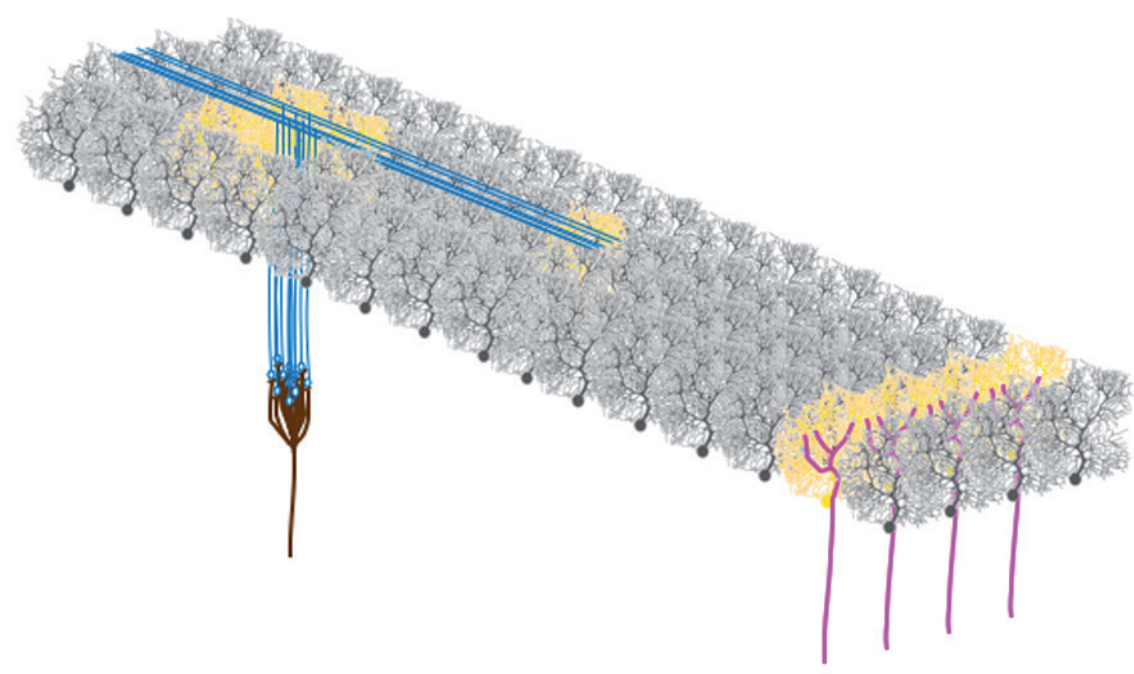

Figure 3 | A schematic representation of the activation of Purkinje cells by mossy fiber and climbing fiber inputs. A mossy fiber input activates a group of granule cells. These, in turn activate the Purkinje cells right about them via synapses along the ascending axon, and may also sparsely activate Purkinje cells via synapses of the parallel fibers. Climbing fibers on the other hand, activate a Purkinje cells that are organized along a parasagittal band. Activated Purkinje cells are depicted in orange.

of the granule cells axons that innervate a given Purkinje cell. The multiple synaptic connections between the ascending axons and Purkinje cells, as well as the synchrony of activation, will maximize the response of this group of Purkinje cells. Purkinje cells that are located laterally along the parallel fibers will be weakly activated not only because of the single en-passant synaptic connections but also because of the non-synchronous volley due to variable conduction velocities of the different parallel fibers. The finding that synaptic junctions along these ascending segments have higher release probability (Sims and Hartell, 2005), accentuates the differences between the two parts of granule cell axons. Furthermore, Isope and Barbour (2002) demonstrated that up to $85 \%$ of the parallel fiber - Purkinje cell synapses do not generate any functional connections. Hence from a functional point of view, the parallel fibers are far less efficient than the ascending portion of the granule cells axon in activating Purkinje cells.

A different mechanistic explanation for the radial organization was proposed by Bower (2002). He suggested that the radial activation of Purkinje cells results from the suppression of on beam Purkinje cells by disynaptic inhibition from the molecular layer interneurons. Santamaria et al. (2007) demonstrated that normally sensory stimulation evokes responses in Purkinje cells in a restricted cerebellar cortical patch, whereas, after blocking inhibitory transmission responses could be recorded at sites that are located distally along the parallel fiber axis. Since the latency of these distal responses is in accordance with the parallel fiber propagation velocity, it has been concluded that they were evoked by parallel fiber input. To reexamine Bower's hypothesis we recorded responses to mossy fiber stimulation both in control conditions and in the presence of the GABA blocker GABAzine, and quantified their spatial organization (Rokni et al., 2008). Our results were in sharp contrast with Santamaria et al. (2007). Although GABAzine significantly prolonged responses to mossy fiber stimulation, it did not change their spatial organization. Namely, propagating beams of Purkinje cell activity did not emerge. The disagreement between our results and those of Santamaria et al. (2007) could be explained, either by the sparse sampling of Purkinje cells by the extracellular electrodes, or by the insensitivity of our imaging system to minute voltage changes. The latter is unlikely as the effects of inhibition blockade within the responding area were easily detected. It is thus reasonable to conclude that the major mechanistic explanation for radial organization of the mossy fiber input resides in the differences between the synapses of the ascending and parallel portions of granule cell axons. 
WHAT ARE THE PARALLEL FIBERS G00D FOR? The radial organization of the mossy fiber input raises a fundamental question: What is the role of parallel fibers in cerebellar function? It seems inconceivable that the parallel fibers, so extremely conserved throughout vertebrate evolution, do not activate Purkinje cells. So why is it that their effect on Purkinje cells cannot be measured? And what is the advantage to be arranged in parallel lines? It is important to stress that the demonstration of the radial organization of the mossy fiber input does not by any means imply that Purkinje cells can never be activated by the parallel fibers in natural conditions. What it implies is that granule cells will mainly activate Purkinje cells via the ascending axon but it does not exclude sparse activation of Purkinje cells along the parallel fibers. This is schematically illustrated in Figure 3. The differences between the ascending axons and the parallel fibers that were outlined above, strongly suggest that there is a fundamental functional difference between them. This is further supported by the recent finding (Sims and Hartell, 2006) that synapses of the ascending axon are resistant to classical forms of parallel fiber - Purkinje cell long term synaptic plasticity (LTD and LTP). It follows from this study that a mossy fiber input mainly activates Purkinje cells via hardwired granule cell Purkinje cell synapses. Additional Purkinje cells that are activated via the parallel fibers could be selected by mechanisms of synaptic plasticity that have been extensively studied (Coesmans et al., 2004; Ito and Kano, 1982; Lev-Ram et al., 2003). Such ability of the system to activate Purkinje cells along the beam following a plasticity protocol has been demonstrated (Jorntell and Ekerot, 2002). Thus the two segments of granule cell axons serve two distinct functions: innate control (ascending) and acquired control (parallel).
The fact that most inputs that drive the principal cells in the cerebellar cortex are predetermined by hardwiring has significant implications for our understanding of cerebellar function. It suggests that the cerebellum should be regarded as a control machine rather than a learning machine. The main part of the control capabilities of behavior is an innate ability that is achieved through synapses along the ascending axons; it is not acquired by learning. Parallel fiber synapses with their plastic capabilities enable fine-tuning of this control system. We propose, therefore, that the anatomical arrangement of the parallel fibers and Purkinje cell dendrites is an efficient packaging, in which the number of potential connections between granule cells and Purkinje cells is maximized. This enables selection and activation of the most appropriate Purkinje cells for any given task.

The cerebellum has commonly been suggested to provide the timing signal necessary for controlling behavior, and this has been supported by many experiments (Braitenberg and Atwood, 1958; Timmann et al., 1999; Welsh and Llinas, 1997), for a review see Ivry and Spencer (2004). As parallel fibers are the most numerous input to Purkinje cells, it seemed probable that they may generate accurate time intervals (Braitenberg and Atwood, 1958). However, as the basic rhythm of the motor system runs around the $10 \mathrm{~Hz}$ frequency, the delay along the parallel fibers cannot play an important role for any practical purposes. The activation of Purkinje cells along the parallel fibers is almost synchronous from the point of view of motor performance. Thus, timing should be accredited to some other part of the system.

\section{ACKNOWLEDGEMENTS}

This work was supported by a BSF grant.

\section{REFERENCES}

Albus, J. S. (1971). A theory of cerebellar function. Math. Biosci. 10, 25-61.

Bell, C. C., and Grimm, R. J. (1969). Discharge properties of Purkinje cells recorded on single and double microelectrodes. J. Neurophysiol. 32, 1044-1055.

Bower, J. M. (2002). The organization of cerebellar cortical circuitry revisited: implications for function. Ann. N.Y. Acad. Sci. 978, 135-155.

Bower, J. M., and Woolston, D. C. (1983). Congruence of spatial organization of tactile projections to granule cell and Purkinje cell layers of cerebellar hemispheres of the albino rat: vertical organization of cerebellar cortex. J. Neurophysiol. 49, 745-766.
Braitenberg, V., and Atwood, R. P. (1958). Morphological observations on the cerebellar cortex. J. Comp. Neurol. 109, 1-33.

Braitenberg, V., Heck, D., and Sultan, F. (1997). The detection and generation of sequences as a key to cerebellar function: experiments and theory. Behav. Brain Sci. 20, 229-245; discussion 245-277.

Brand, S., Dahl, A. L., and Mugnaini, E. (1976). The length of parallel fibers in the cat cerebellar cortex. An experimental light and electron microscopic study. Exp. Brain Res. 26, 39-58.

Chen, G., Hanson, C. L., and Ebner, T. J. (1998). Optical responses evoked by cerebellar surface stimulation in vivo using neutral red. Neuroscience 84, 645-668.
Coesmans, M., Weber,J.T., De Zeeuw, C. I., and Hansel, C. (2004). Bidirectional parallel fiber plasticity in the cerebellum under climbing fiber control. Neuron 44, 691-700.

Cohen, D., and Yarom, Y. (1998). Patches of synchronized activity in the cerebellar cortex evoked by mossy-fiber stimulation: questioning the role of parallel fibers. Proc. Natl. Acad. Sci. U.S.A. 95, 15032-15036.

Cohen, D., and Yarom, Y. (1999). Optical measurements of synchronized activity in isolated mammalian cerebellum. Neuroscience 94, 859-866.

Cohen, D., and Yarom, Y. (2000). Cerebellar on-beam and lateral inhibition: two functionally distinct circuits. J. Neurophysiol. 83, 1932-1940.
Ebner, T. J., and Bloedel, J. R. (1981). Correlation between activity of Purkinje cells and its modification by natural peripheral stimuli. J. Neurophysiol. 45, 948-961.

Eccles, J. C., Llinas, R., and Sasaki, K. (1966). Parallel fibre stimulation and the responses induced thereby in the Purkinje cells of the cerebellum. Exp. Brain Res. 1, 17-39.

Eccles, J. C., Sabah, N. H., Schmidt, R. F., and Taborikova, H. (1972). Cutaneous mechanoreceptors influencing impulse discharges in cerebellar cortex. II. In Purkyne cells by mossy fiber input. Exp. Brain Res. 15, 261-277.

Garwicz, M., and Andersson, G. (1992). Spread of synaptic activity along paral- 
lel fibres in cat cerebellar anterior lobe. Exp. Brain Res. 88, 615-622.

Harvey, R. J., and Napper, R. M. (1988). Quantitative study of granule and Purkinje cells in the cerebellar cortex of the rat. J. Comp. Neurol. 274, 151-157.

Heck, D.H., Thach, W.T., and Keating, J. G. (2007). On-beam synchrony in the cerebellum as the mechanism for the timing and coordination of movement. Proc. Natl. Acad. Sci. U.S.A. 104, 7658-7663.

Isope, P., and Barbour, B. (2002). Properties of unitary granule cell $\rightarrow$ Purkinje cell synapses in adult rat cerebellar slices. J. Neurosci. 22, 9668-9678.

Ito, M., and Kano, M. (1982). Long-lasting depression of parallel fiber-Purkinje cell transmission induced by conjunctive stimulation of parallel fibers and climbing fibers in the cerebellar cortex. Neurosci. Lett. 33, 253-258.

Ivry, R. B., and Spencer, R. M. (2004). The neural representation of time. Curr. Opin. Neurobiol. 14, 225-232.

Jorntell, H., and Ekerot, C. F. (2002). Reciprocal bidirectional plasticity of parallel fiber receptive fields in cerebellar Purkinje cells and their afferent interneurons. Neuron 34, 797-806.

Lev-Ram, V.,Mehta,S. B., Kleinfeld,D., and Tsien, R.Y. (2003). Reversing cerebellar long-term depression. Proc. Natl. Acad. Sci. U.S.A. 100, 15989-15993.

Llinas, R. (1982). General discussion: radial connectivity in the cerebellar cortex: a novel view regarding the functional organization of the molecular layer. In The Cerebellum: New Vistas, S.L. Palay and V.Chan-Palay, ed. (New York, Springer), pp. 189-192.

Llinas, R., Yarom, Y., and Sugimori, M. (1981). Isolated mammalian brain in vitro: new technique for analysis of electrical activity of neuronal circuit function. Fed. Proc. 40, 2240-2245.

Mann-Metzer, P., and Yarom, Y. (1999). Electrotonic coupling interacts with intrinsic properties to generate synchronized activity in cerebellar networks of inhibitory interneurons. J. Neurosci. 19, 3298-3306.

Marr, D. (1969). A theory of cerebellar cortex. J. Physiol. 202, 437-470.

Mugnaini,E.(1983). The length of cerebellar parallel fibers in chicken and rhesus monkey. J. Comp. Neurol. 220, 7-15.

Napper, R. M., and Harvey, R. J. (1988). Number of parallel fiber synapses on an individual Purkinje cell in the cerebellum of the rat. J. Comp. Neurol. 274, 168-177.

Ohyama, T., Nores, W. L., Murphy, M., and Mauk, M. D. (2003). What the cerebellum computes. Trends Neurosci. 26, 222-227.

Palay, S. L., and Chan-Palay, V. (1974). Cerebellar Cortex, Cytology and Organization. Berlin, Springer-Verlag.

Rokni, D., Llinas, R., and Yarom, Y. (2008). Stars and stripes in the cerebellar cortex: a voltage sensitive dye study. Front. Syst. Neurosci. 1, 1.
Santamaria, F., Tripp, P. G., and Bower, J. M. (2007). Feedforward inhibition controls the spread of granule cell-induced Purkinje cell activity in the cerebellar cortex. J. Neurophysiol. 97, 248-263.

Shambes, G. M., Beermann, D. H., and Welker, W. (1978). Multiple tactile areas in cerebellar cortex: another patchy cutaneous projection to granule cell columns in rats. Brain Res. 157 123-128.

Shinoda, Y., Sugihara, I., Wu, H. S., and Sugiuchi, Y. (2000). The entire trajectory of single climbing and mossy fibers in the cerebellar nuclei and cortex Prog. Brain Res. 124, 173-186.

Sims, R. E., and Hartell, N. A. (2005). Differences in transmission properties and susceptibility to long-term depression reveal functional specialization of ascending axon and parallel fiber synapses to Purkinje cells. $J$. Neurosci. 25, 3246-3257.

Sims, R. E., and Hartell, N. A. (2006). Differential susceptibility to synaptic plasticity reveals a functional specialization of ascending axon and parallel fiber synapses to cerebellar Purkinje cells. J. Neurosci. 26, 5153-5159.

Sotelo, C., and Llinas, R. (1972).Specialized membranejunctions between neurons in the vertebrate cerebellar cortex. J. Cell Biol. 53, 271-289.

Thach, W. T., Goodkin, H. P., and Keating, J. G. (1992). The cerebellum and the adaptive coordination of movement. Annu. Rev. Neurosci. $15,403-442$.

Timmann, D., Watts, S., and Hore, J. (1999). Failure of cerebellar patients to time finger opening precisely causes ball high-low inaccuracy in overarm throws. J. Neurophysiol. 82, 103-114.

Welsh, J. P., and Llinas, R. (1997). Some organizing principles for the control of movement based on olivocerebellar physiology. Prog. Brain Res. 114 449-461.

Wolpert, D. M., Miall, R. C., and Kawato, M. (1998). Internal models in the cerebellum. Trends Cogn. Sci. 2, 338-347.

Conflict of Interest Statement: The authors declare that the research was conducted in the absence of any commercial or financial relationships that could be construed as a potential conflict of interest.

Received: 29 September 2008; accepted: 10 November 2008; published: 15 December 2008.

Citation: Front. Neurosci. (2008) 2, 2:192 198. doi: 10.3389/neuro.01.036.2008 Copyright: (๑) 2008 Rokni, Llinas and Yarom. This is an open-access article subject to an exclusive license agreement between the authors and the Frontiers Research Foundation, which permits unrestricted use, distribution, and reproduction in any medium, provided the original authors and source are credited. 